\title{
Cursor trajectory analysis
}

\author{
H. Keuning-Van Oirschot, A.J.M. Houtsma \\ IPO, Center for User-System Interaction \\ P.O. Box 513 \\ 5600 MB Eindhoven \\ The Netherlands \\ tel: +31402475210 \\ h.keuning@tue.nl
}

Eindhoven University of Technology, August 4, 2000

\section{Introduction}

As computers evolve to be basic tools in work and home, improving the human-computer interaction is critical for the user's acceptance. Among the criteria that can be listed, a system must be comfortable and efficient. This means, for example, that a computer must give the user fast and clear feedback on his or her actions. One way is by giving a sensation of touch to the moving hand via the control device. In controlled experimental environments tactual feedback devices, indeed, turned out to facilitate the user's target acquisition task (Keyson, 1997; Akamatsu and Sato, 1994; Göbel et al., 1995). In these experimental environments, however, only one visual object was instructed as being the target. So, there was only one object which could activate the touch feedback mechanism. In typical human computer interaction several objects are on the screen which are non-targets, but which still activate the touch feedback mechanism when entered. In such an environment with non-targets, it would be more convenient and effective if the feedback works for the user's target only. This is not possible, as long as the program doesn't know where the user wants to go. The obvious solution is to predict the user's goal. After predicting the target the computer system can aid the user to reach the target without getting distracted by touch feedback on non-targets.

\section{Purpose of this experiment}

The general purpose of this study is to make a prediction algorithm based on characteristics of the initial part of the cursor trajectory. Therefore, it is necessary to know what trajectory characteristics can give useful information for a reliable target prediction. One aspect of a trajectory is its path. This is the spatial time-independent shape of the trajectory. The other aspect is the time dependence along the path (Hollerbach, 1990).

In the first experiment we focused on the path. We investigated what the movement paths look like in different directions and with different devices. The main questions were, whether a constant curve could be detected and whether curvature and/or its variability related to direction, device or user.

\section{Procedure}

The task of the subjects was to move the cursor to a certain target, in one of eight directions, using a certain control device. The direction from the starting point to the targets was horizontal, vertical or diagonal. So, a target could be projected in every 45 degree angle. As all targets were at the same distance and had the same width, the index of difficulty (Fitts, 1954) was the same for each trial. This was done, because 'direction' and 'device' were planned to be the only within subject factors in this first exploratory experiment. The control device could be a mechanical mouse an optical mouse or an optical trackball.

Participants could start whenever they were ready for it. In the middle of the screen a 'start' button was shown (see fig 1 . When pressing the space bar, while the cursor was on the start button, a black circle (one of the eight possible targets) appeared and the button disappeared. Meanwhile the cursor was repositioned on specified coordinates, so that each trial started exactly at the same position. The 


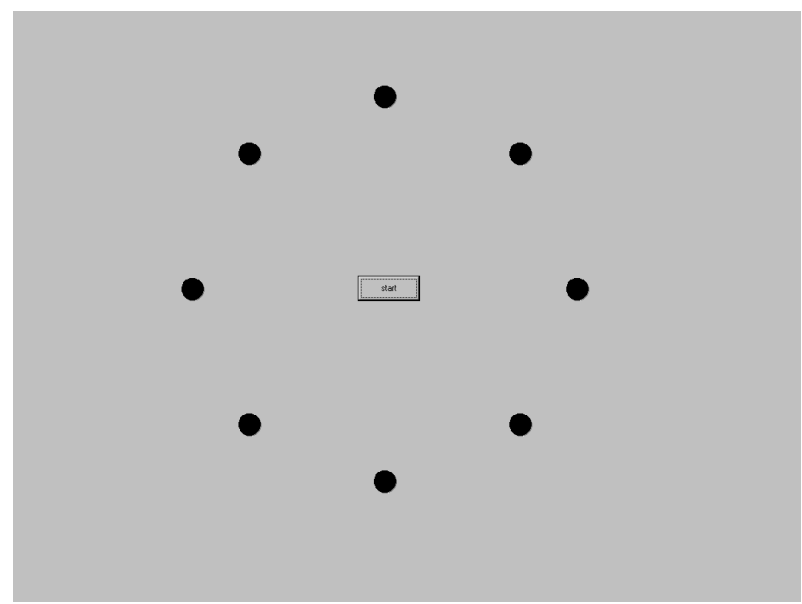

Figure 1: All possible targets

participants were instructed to reach the target in a 'normal' way, as they were used to in working an a computer. When the target was reached subjects had to wait 200 msec until the target disappeared and the 'start' button re-appeared. Then the cursor could be moved to the start button again. After pressing the space bar the next trial started.

\section{Design}

The experiment was divided in three sessions, each with one of three control devices, a normal mouse, an optical mouse or an optical trackball. The order in which the devices were used was random. Each session consisted of a practice and an experimental part. In the experimental part each target was presented ten times, randomly.

\section{Measurements}

During each trial $\mathrm{x}$ - and $\mathrm{y}$-coordinates (in pixels) and the system time were sampled. This was done at $50 \mathrm{~Hz}$, because the input devices had a default frequency of $50 \mathrm{~Hz}$.

\section{Subjects}

Subjects were ten employees at IPO. Seven male, three female. Mean age was 29.8 years. All of them were experienced with a mechanical mouse, but not with an optical mouse or trackball.

\section{Analysis}

Because sampling was time controlled, velocity influences the spacing between the samples. To calculate the curvature of a path, however, we want equal distances between the samples, because then every part of the path contributes equally to the calculation of the curvature. Therefore, for each trajectory, new samples were calculated, by interpolation, for every $1 \%$ of total traveled distance (distance of the path itself).

To assess the curvature, the paths were first rotated until start and end positions were on the positive $\mathrm{x}$-axis. Then the distance between each calculated coordinate and the $\mathrm{x}$-axis was taken, which is equal to the accompanying y-coordinate, both with respect to value and sign. Curvature was then defined as the mean value of this distance along the path (Boessekool, 1999). The variability of the paths is represented by the standard deviation of all sample points around the mean path of the ten trajectories performed per person per direction and device.

The standard deviation is calculated over each 10percent section of the whole path.

\section{Results}

Figure 2 shows the mean paths and standard deviation in every direction over the three devices.

It can be seen that the mean paths are rather

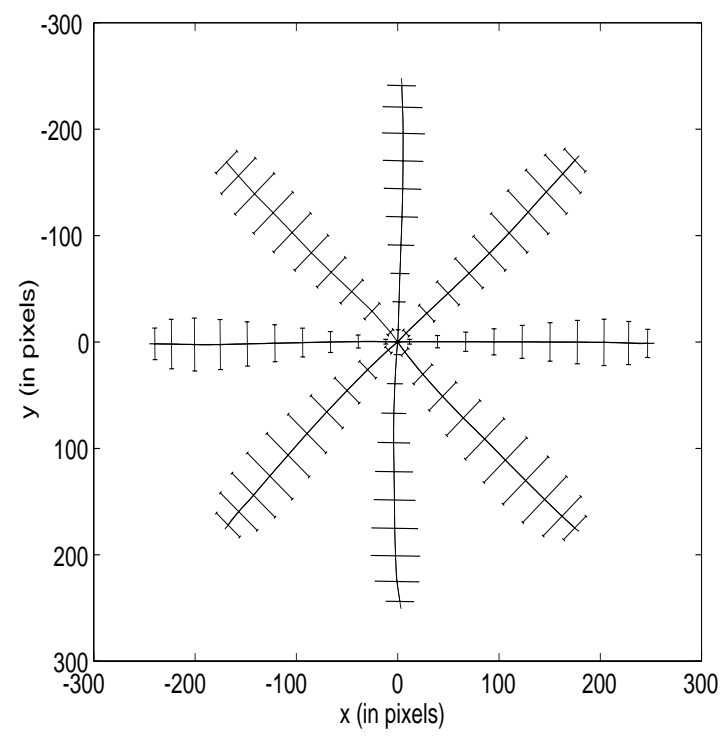

Figure 2: Mean path and st.dev. for all three devices (mechanical mouse, optical mouse and optical trackball) in eight directions.

straight. A statistical t-test of the mean curvature against a curvature of zero shows that the overall paths are slightly but significantly curved $(\mathrm{p}<.01)$. Figure 3 shows the same as figure 2 , 

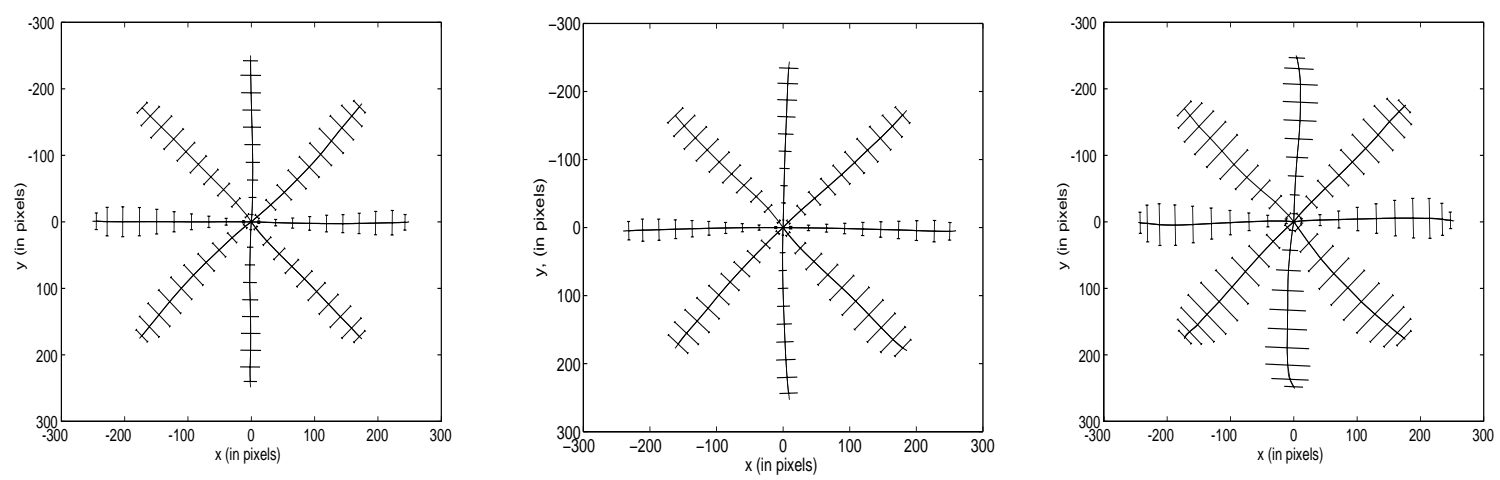

Figure 3: Mean path and st.dev. for three devices a)mechanical mouse, b)optical mouse and c)optical trackball in eight directions.

but for each device separately. Only the southward paths created with the optical mouse anc the optical trackball are visibly curved. A nonparametric test(Friedman test) was performed pe1 device to test for differences in curvature betweer direction. This test showed that only for the optical mouse a difference in curvature between directions existed $\left(\chi^{2}=15.4, p=.031\right)$. More specified it showed that moving in a vertical directior toward the body (southward on a compass card' resulted in a high ranking score. With a repeatec measure ANOVA also, a significant effect of 'user was found $\left(F_{1,9}=13.245, p=.005\right)$. Another two ANOVA's per user on device and direction, respectively, showed that some users were influenced by the device; especially the optical trackball resulted in more curved paths.

Obviously, most variability is present at the paths created with the optical trackball. It also shows that the standard deviations do not overlap. This means that with this target resolution every intended target should be highly predictable. With a prediction requirement of $69 \%$ correct (i.e. an angular target separation of one standard deviation)then the resolution could be about 30 equally-spaced targets for the two mice and about 20-25 for the optical trackball.

Figure 4 shows variability as a function of direction pooled over all subjects and sample points. It can be seen that the optical mouse leads to the least variability around the mean paths and the optical trackball to the most variability. Also, the oblique directions (ne, se, sw and nw) show higher standard deviations than the horizontal and vertical paths. This can be seen in figure 2 and 3 as well.

To test for differences in variability between

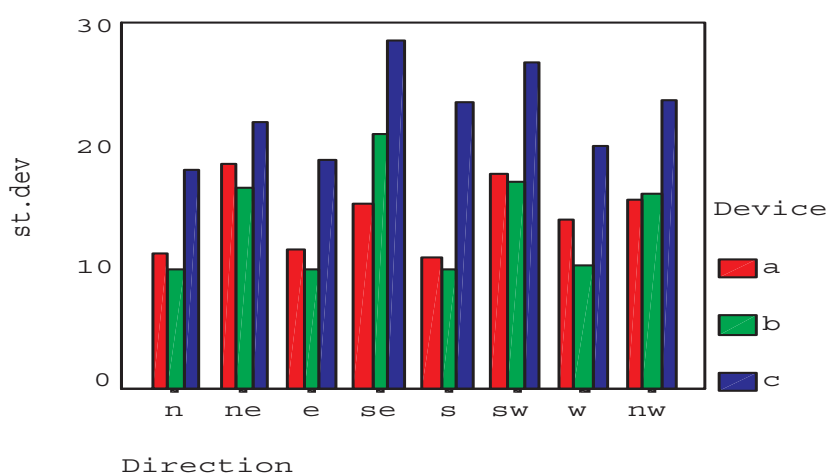

Figure 4: Mean variability per direction for each device a) mechanical mouse, b) optical mouse, c) optical trackball.

direction and device, a repeated measure ANOVA was performed with 'direction' and 'device' as within subject factors and 'user' as between subjects factor. Two main effects were found: for device $\left(F_{2,18}=22.938, p=<.01\right)$, direction $\left(F_{7,63}=13.243, p<.01\right)$, as well as an interaction effect between device and direction $\left(F_{14,126}=3.918, p<.01\right)$. Also an effect of 'user' $\left(F_{1,9}=147.029, p<.01\right)$ was found.

\section{Conclusions}

- Although there is a statistical significant curvature (as opposed to straightness) in some of the measured paths, the mean paths appear rather straight in comparison with standard deviations. For target estimation purposes only negligible losses are to be 
expected if paths are assumed to be straight lines.

- Variability around the mean path differs per device and per direction. This means that for a good prediction the target resolution could be higher when the device creates less variable paths (e.g. the optical mouse or the mechanical mouse).

- Variability also differs per 'user'. This implies that a target estimation algorithm could be further improved by adjusting its parameters beforehand to individual users.

\section{References}

Akamatsu, M. and S. Sato (1994). A multi-modal mouse with tactile and force feedback. Int. $J$. Human-Computer Studies 40, 443-453.

Boessekool, J. (1999). Moving two hands. Ph. D. thesis, Utrecht University, the Netherlands.

Fitts, P. (1954). The information capacity of the human motor system in controlling the amplitude of movement. Journal of Experimental Psychology 47(6), 381-391.

Göbel, M., H. Luczak, J. Springer, V. Hedicke, and M. Rötting (1995). Tactile feedback applied to computer mice. International Journal of Human-Computer Interaction 7(1), 1-24.

Hollerbach, J. (1990). Planning of arm movements. In: K. S. H. J. Osherson, D.N. (Ed.), Visual cognition and action (pp183211). Cambridge, MA:MIT Press.

Keyson, D. (1997). Touch in user interface navigation. Ph. D. thesis, Technical University Eindhoven, The Netherlands. 